\title{
El dominio de competencias transversales en Educación Superior en diferentes contextos formativos ${ }^{1}$
}

\author{
Pilar Martínez Clares² \\ http://orcid.org/0000-0002-5649-931X \\ Natalia González Morga² \\ https://orcid.org/0000-0002-5544-4464
}

\section{Resumen}

La alternancia de contextos en la formación universitaria alude a las múltiples experiencias académicas y profesionales que combinan los estudiantes a lo largo de su trayectoria universitaria y que pueden complementar la formación basada en competencias impartida en las aulas. Desde este trabajo se pretende indagar en el nivel de dominio del estudiante de grado en competencias transversales en relación a distintas variables curriculares (asistencia a clase, nota del expediente o horas de estudio a la semana) y extracurriculares (participación en actividades universitarias, becas de formación, investigación o movilidad, situación laboral). Para ello, han participado en la investigación 1137 estudiantes de último año de grado de las cinco ramas de conocimiento (Ciencias Sociales, Ciencias de la Salud, Ciencias Experimentales, Artes y Humanidades e Ingenierías), por medio de un cuestionario diseñado ad hoc. Entre los resultados destaca un dominio aceptable, aunque mejorable, de las competencias transversales en los futuros egresados, siendo la competencia más dominada el compromiso ético y social y el trabajo en equipo y la menos dominada, la lengua extranjera. Se constata una relación significativa y positiva entre la nota del expediente académico o las horas dedicadas al estudio con el incremento del nivel de dominio, de igual manera que con la alternancia de actividades universitarias, becas de formación, investigación o de movilidad y de trabajo durante la carrera. Estos resultados señalan las posibles líneas de actuación para potenciar la adquisición de las competencias transversales en las aulas universitarias y, en consecuencia, mejorar la empleabilidad de los egresados.

\section{Palabras clave}

Competencias - Educación superior - Contextos de aprendizaje - Formación.

\footnotetext{
1- Esta investigación pertenece a un estudio más amplio titulado Un estudio de competencias transversales en la Universidad de Murcia financiado por el MECD dentro del Programa Nacional de Formación de Recursos Humanos de Investigación (FPU) en el marco del Plan Nacional de Investigación Científica, Desarrollo e Innovación Tecnológica 2008-2011. nº AP-04112.

2- Universidad de Murcia, Murcia, España. Contactos: pmclares@um.es; natalia.gonzalez@um.es.
}

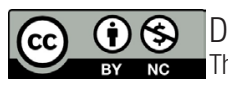

DOl: http://dx.doi.org/10.1590/S1678-4634201945188436

This content is licensed under a Creative Commons attribution-type BY-NC. 


\section{The domain of transversal competences in Higher Education in different training contexts}

\section{Abstract}

The alternation of contexts in university education refers to the multiple academic and professional experiences that students combine throughout their path in higher education. These can complement the competency-based training provided in the classroom. This article aims to investigate the level of domain of the grad students in transversal competences in relation to different variables both curricular (class attendance, file records. or weekly hours of study) and extracurricular (participation in university activities, training scholarships, research or mobility, employment situation). For such, 1137 senior students from the five areas of knowledge (Social Sciences, Health Sciences, Experimental Sciences, Arts and Humanities, and Engineering), participated in the study by answering a questionnaire designed ad hoc. The results include an acceptable, although improbable, domain of transversal competences in future graduates, with the most dominated competence being the ethical and social commitment and teamwork, and the least dominated, the foreign language. There is a significant and positive relationship between the file record of grades or the hours dedicated to the study and the increased level of domain, similar to the alternation of university activities, training scholarships, research or mobility, and work along the career. These results indicate the possible lines of action to increase the achievement of transversal competences in higher-education classrooms and, consequently, improve the employability of graduates.

\section{Keywords}

Competences - Higher education - Learning contexts - Training.

\section{Introducción}

El modelo socioeconómico en el que se ubica la Educación Superior del siglo XXI se caracteriza por la abundancia de información, la rápida caducidad del conocimiento, la fragmentación de fronteras entre países, las múltiples interconexiones y circularidad de una economía en continuo reciclaje. Cada uno de estos aspectos supone un punto de inflexión en el diseño curricular de la formación de los egresados con la pretensión de afrontar la constante que subyace a todos ellos: impulsar, como nunca antes, el desarrollo de una cultura de aprendizaje que permita estar capacitado para afrontar la presión de los cambios sucesivos que estos fenómenos ocasionan en todos los ámbitos vitales.

En esta complejidad social, no es suficiente que los ciudadanos tengan una formación especializada en diferentes ocupaciones, sino que es necesario que la dimensión más personal 
y social esté presente para evitar abordar los problemas de forma fragmentada y desde una única perspectiva técnica o cognitiva. Las actuaciones eficaces se alcanzan cuando se consigue movilizar las diferentes dimensiones y recursos de la persona en una misma dirección, de sumar esfuerzos, rasgos y capacidades. Es precisamente en este aspecto donde radica la importancia de una formación integradora e integral en las universidades, que fomente y potencie paralelamente competencias técnicas relacionadas con campos de estudio particulares y competencias más sociales, personales y emocionales de amplio alcance a distintas tareas y situaciones. Estas últimas competencias son las denominadas transversales y tienen un amplio rango de funciones y aplicaciones que desbordan las barreras disciplinarias de gran valor para un modelo social y productivo en permanente cambio.

La capacidad de adaptación no es una situación para la que se nace preparado, requiere de una buena predisposición al aprendizaje a lo largo de la vida y de su consecuente dedicación a conformar una serie de habilidades, destrezas, actitudes y valores que aporten flexibilidad a nuestra forma de entender la realidad y eficacia a la hora de abordarla. Tener un bagaje amplio de competencias transversales proporciona estas cualidades, lo que justifica la inversión de tiempo y esfuerzo desde las instituciones universitarias para su inclusión curricular desde la implantación del Espacio Europeo de Educación Superior (EEES). La formación tiene que reunir los aspectos clave para la realidad actual que para Villa y Poblete (2011) son: flexibilidad, movilidad y transferibilidad.

Hoy no se entiende una formación sin estas competencias, ya que son las que proporcionan al estudiante una disposición a cooperar, comunicar, tomar decisiones y crear e innovar entre otras destrezas clave y conforman un perfil académico-profesional flexible, polivalente y con capacidad de adaptación continua, tal y como requiere el actual ámbito sociolaboral. La transición de la sociedad del conocimiento hacia la sociedad del aprendizaje o el surgimiento, según los últimos informes del Foro Económico Mundial, de la 4ª Revolución Industrial (WFE, 2016; SCHWAB, 2016), crean nuevos puestos de trabajo, contenidos, medios, métodos y formas de trabajo que configuran un nuevo concepto de profesionalidad, que demanda cualificaciones y competencias en esta dirección. Cobo y Moravec definen a este nuevo profesional como "[...] un trabajador innovador, imaginativo, creativo, capaz de trabajar con prácticamente cualquier persona, en cualquier lugar y en cualquier momento" (2011, p. 56).

Ante este escenario, no es de extrañar que distintos informes internacionales consideren a las competencias transversales las más demandas en el mercado laboral (CEDEFOP, 2014; HUMBERG; VAN DER VALDEN; VERHAGEN, 2013; OIT, 2015), subrayando, a la vez, que una formación universitaria que pasa por alto las competencias transversales es incompleta e insuficiente para obtener resultados óptimos en el futuro desarrollo personal y profesional del estudiante. Sus propias características de transferibilidad, multifuncionalidad y multidimensionalidad las hacen esenciales en el actual momento socioeconómico (RYCHEN; SALGANIK, 2003), pero también complejas a la hora de adquirirlas.

$\mathrm{Su}$ carácter genérico les atribuye distintas posibilidades de desarrollo y formas de aprenderlas que desbordan a las instituciones educativas; por todo ello, pensar en responsabilizar a las universidades del nivel de dominio del egresado, es una reflexión 
simplista, que en ningún caso puede explicarse por importante que esta institución sea (PLANAS COLL; ENCISO ÁVILA, 2014). Las competencias, difícilmente desligadas de la acción o puesta en práctica, no solo se desarrollan y se adquieren como parte de la educación formal recibida a través del contenido de las asignaturas, sino en cualquier situación de aprendizaje o formación a lo largo de la vida. La nueva arquitectura de la educación entiende el aprendizaje como un continuo que se prolonga durante la vida y que puede ocurrir en cualquier momento o lugar (COBO; MORAVEC, 2011). Este enfoque amplía la adquisición de competencias transversales a ámbitos que van más allá de los curriculares, y propone tener en cuenta la repercusión del aprendizaje no formal e informal.

A lo largo de este trabajo, se pretende conocer el nivel de dominio de las competencias transversales del estudiante y su relación con la experiencia académica y profesional vivenciada durante los estudios universitarios para determinar sus factores más influyentes. Se contrastan distintas variables referidas a la experiencia académica como: asistencia a clase, horas de estudio y nota media, y otras sobre experiencias extracurriculares procedentes, bien de haber disfrutado de becas de formación, movilidad o investigación, o bien, de realizar otras actividades universitarias paralelas al currículo o derivadas del ámbito profesional, como el trabajo remunerado. Los resultados obtenidos son una buena fuente para reflexionar sobre la acción a seguir a fin de obtener una formación en el egresado de mayor pertinencia, capaz de reducir la brecha entre aquello que se enseña y se aprende desde las universidades y lo que demanda el mundo sociolaboral en el futuro de la Industria 4.0, entendida como la cuarta revolución laboral marcada por la digitalización y el avance vertiginoso del conocimiento.

\section{La alternancia de contextos y el dominio de las competencias transversales}

El concepto de alternancia de contextos en la Educación Superior hace referencia a las múltiples realidades que experimentan y vivencian los estudiantes de forma más o menos intensa a lo largo de su trayectoria universitaria, a partir de las cuales se obtienen experiencias que pueden sumar tácitamente competencias o complementar las adquiridas en la formación que se imparte en las aulas universitarias.

Estas modalidades de formación institucionalizadas aluden a los “[...] periodos de formación que los estudiantes universitarios realizan fuera de la universidad en contextos profesionales reales", así define Zabalza (2013, p. 19) las prácticas externas, o bien, a las actividades culturales, de representación estudiantil y deportivas que alternan con los estudios; el periodo de tiempo que invierten en otras universidades como resultado de la participación en programas o proyectos internacionales educativos, o el hecho de combinar estudios con trabajo. Esta última situación ha crecido de forma constante en los últimos años (FINKEL; BARAÑANO, 2014) y junto con el valor de mantener el contacto permanente con la realidad profesional para la asimilación de las competencias, se convierte en otra posible modalidad de formación, en este caso sin planificación ni intencionalidad formativa por parte de las universidades.

Igualmente, como consecuencia de los procesos de globalización y del desarrollo del EEES, los procesos de internacionalización de las universidades han ido intensificándose. La 
modernización de las universidades persigue que estas instituiciones estén académicamente más preparadas, sean más eficientes y estén más internacionalizadas para proyectar hacia el futuro. Con esta finalidad se propone un conjunto de medidas orientadas a aumentar el atractivo internacional de las universidades entre las que destacan la participación en proyectos y programas internacionales educativos como Erasmus.

Los efectos de los programas de internacionalización educativa en el desarrollo de las competencias transversales cuentan ya con sus primeras evidencias. Rodríguez Izquierdo (2015) indagó sobre su potencialidad como estrategia pedagógica para la adquisición de competencias transversales y los resultados confirman su eficacia en la adquisición de aquellas de carácter interpersonal. Bajo estas premisas teóricas se aprecia una cierta relación de causalidad, que sin ser lineal debido a que otros factores también inciden en el dominio de estas competencias, cumplen un papel relevante que no se debe pasar por alto en la formación universitaria y sobre el que pretende profundizar en este trabajo. Entre esos otros elementos influyentes se encuentran las materias curriculares con un carácter más dinámico e interactivo con el entorno, como son los créditos de libre configuración y las prácticas externas.

Los planes de estudio de los títulos de grado contemplan créditos de libre configuración o también denominados créditos CRAU (Créditos para la Realización de Actividades Universitarias) en el contexto de este trabajo, referidos a una serie de actividades culturales, deportivas, de solidaridad y cooperación (entre las que se encuentra las prácticas externas y el voluntariado), así como de representación estudiantil, que promueven de forma paralela al currículo universitario y cuya realización es totalmente voluntaria y de libre elección. Los beneficios de estos créditos han sido escasamente analizados, sin embargo, su alto dinamismo y activismo, los hace especialmente importantes y coherentes con una formación basada en competencias que lleva a replantear sus oportunidades en el dominio de las denominadas competencias transversales.

Levy Leboyer hace ya dos décadas afirmó que “[...] las experiencias obtenidas de la acción, de la asunción de responsabilidad real y del enfrentamiento a problemas concretos, aportan realmente competencias que la mejor enseñanza jamás será capaz de proporcionar" (1997, p. 27), poniendo de manifiesto la superioridad de la formación en el contexto respecto a otros medios. De forma análoga, Le Boterf también manifestó que "[...] si las competencias son indisociables de su puesta en marcha, su ejercicio es necesario para que se mantengan. Las averías, los incidentes, los problemas o los proyectos son oportunidades necesarias para el mantenimiento y el desarrollo de las competencias" (1995, p. 18). De este modo, los procesos de aprendizaje que generen el paradigma pedagógico basado en competencias deben orientarse hacia la acción del participante, hacia la práctica y la interacción con el medio, “[...] tomando como referente el marco organizativo en el que la situación de trabajo es situación de aprendizaje” (TEJADA, 2012, p. 20). De ahí que toda experiencia o contacto con el entorno socioprofesional, esté o no programada e institucionalizada por las universidades, pueda ser fuente de adquisición y desarrollo de competencias y factor de profesionalización.

Estos argumentos sitúan a esta investigación en la necesidad de reflexionar también sobre la pertinencia de estar empleado durante los estudios para la adquisición 
de competencias dado el constante crecimiento de este fenómeno en las universidades españolas. El informe Panorama de la Educación: indicadores de la Organización para la Cooperación y el Desarrollo Económicos (OCDE, 2013) y la Encuesta de condiciones de vida y participación de los estudiantes universitarios (ECOVIPEN) (ARIÑO; LLOPIS; SOLER, 2012), constituyen una primera fuente de datos sobre el colectivo de jóvenes que compatibiliza estudios con trabajo, si bien los estudios sobre las condiciones de vida y participación de los estudiantes universitarios no son muy frecuentes, por lo que se entiende que la preocupación por esta temática es bastante reciente (FINKEL; BARAÑANO, 2014). Un primer dato obtenido del contraste de estas investigaciones realizadas en el mismo año (2011), constata un 54\% de estudiantes dedicados a tiempo completo a los estudios, de forma que poco menos de la mitad compagina estudios con algún tipo de trabajo remunerado.

Algunos países europeos como Dinamarca u Holanda resaltan la importancia de trabajar durante los estudios en su proyección profesional y apoyan desde el sistema educativo el trabajo a tiempo parcial de sus estudiantes. Entienden que esta modalidad de trabajo puede tener beneficios en los estudiantes de cara a poner un punto de partida en su carrera profesional, al tiempo que sirve para contener el desempleo juvenil (NILSSON, 2015; CROWLEY et al., 2013). En EEUU, mientras continúa el debate sobre las ventajas y los inconvenientes de que los estudiantes trabajen, existe una tendencia a favor de combinar ambas actividades desde la óptica del dominio de las competencias. El contacto con el contexto sociolaboral proporciona competencias adicionales decisivas para la empleabilidad que en ocasiones pasan desapercibidas en la formación universitaria y con especial relevancia en el acceso y permanencia en la vida profesional.

A modo de conclusión, con esta investigación se pretende comprobar la relación que existe entre estas y otras variables comentadas a lo largo de este apartado, con el dominio de las competencias transversales. La finalidad que se persigue es acortar distancias entre la universidad y las demandas formativas del ecosistema socioempresarial a partir de propuestas de mejora y readaptación de los títulos, fundamentadas en datos empíricos. La queja de los empleadores sobre el desajuste competencial de los egresados es extensible a todas las regiones y universidades y la necesidad de conseguir la mejora e incremento de la funcionalidad, satisfacción y calidad de la formación de los egresados, además de contribuir a dar un paso más en la transferencia de sus perfiles formativos a perfiles profesionales, es una necesidad cada vez más evidente que apunta a la integración de las competencias transversales en los currículos universitarios y consecuente adquisición por parte del estudiante como la principal opción estratégica. Esta y otras investigaciones muestran que los estudios sobre competencias transversales en la Educación Superior, más que ser una cuestión de moda, son un tema de necesidad con una prioridad inmediata.

\section{Método}

\section{Participantes}

La población objeto de estudio corresponde al alumnado de $4^{\circ}$ de grado de la Universidad de Murcia. La selección de los participantes se realiza a través de un muestreo 
aleatorio estratificado con el que se obtiene una muestra total de 1137 estudiantes, representativa del alumnado de último curso de grado de dicha Universidad (nivel de confianza de 99\% y error muestral de 3,44\%) y distribuidos en diferentes ramas de conocimiento respetando la proporcionalidad del conjunto de los estratos de la población global como representa la tabla 1. Del total de los estudiantes que responden al cuestionario (participantes), el 68\% son mujeres y el 31\% restante hombres y la edad media de todos ellos se sitúa en 23 años.

Tabla 1 - Distribución de los participantes por título y rama de conocimiento y estratificación de la muestra

\begin{tabular}{|c|c|c|c|c|c|}
\hline Rama de conocimiento & $\begin{array}{l}\text { Títulos de grado } \\
\text { participantes }\end{array}$ & $\begin{array}{c}\mathrm{N}^{0} \text { de } \\
\text { participantes }\end{array}$ & $\begin{array}{c}\text { Total de } \\
\text { participantes por } \\
\text { rama }\end{array}$ & $\begin{array}{c}\% \text { de muestra } \\
\text { estratificada } \\
\text { recomendada en } \\
n=1137\end{array}$ & $\begin{array}{c}\text { \% de muestra } \\
\text { estratificada } \\
\text { alcanzada }\end{array}$ \\
\hline \multirow{4}{*}{$\begin{array}{c}\text { Ciencias Sociales } \\
\text { (CSS) }\end{array}$} & Pedagogía & 78 & \multirow{4}{*}{565} & \multirow{4}{*}{$54 \%$} & \multirow{4}{*}{$50 \%$} \\
\hline & Educación Social & 62 & & & \\
\hline & Educación Primaria & 313 & & & \\
\hline & Educación Infantil & 112 & & & \\
\hline \multirow{3}{*}{$\begin{array}{l}\text { Ciencias de la Salud } \\
\text { (CS) }\end{array}$} & Logopedia & 28 & \multirow{3}{*}{181} & \multirow{3}{*}{$18 \%$} & \multirow{3}{*}{$16 \%$} \\
\hline & Enfermería & 98 & & & \\
\hline & Psicología & 55 & & & \\
\hline \multirow{5}{*}{$\begin{array}{l}\text { Ciencias Experimentales } \\
\text { (CE) }\end{array}$} & Ciencias Ambientales & 43 & \multirow{5}{*}{159} & \multirow{5}{*}{$9 \%$} & \multirow{5}{*}{$14 \%$} \\
\hline & Biología & 40 & & & \\
\hline & Física & 16 & & & \\
\hline & Bioquímica & 52 & & & \\
\hline & Matemáticas & 8 & & & \\
\hline \multirow{6}{*}{$\begin{array}{l}\text { Artes y humanidades } \\
\text { (AH) }\end{array}$} & Historia del Arte & 26 & \multirow{6}{*}{181} & \multirow{6}{*}{$17 \%$} & \multirow{6}{*}{$16 \%$} \\
\hline & Geografía & 28 & & & \\
\hline & Traducción Francés & 10 & & & \\
\hline & Traducción Inglés & 26 & & & \\
\hline & Estudios Ingleses & 38 & & & \\
\hline & Historia & 53 & & & \\
\hline $\begin{array}{l}\text { Ingenierías } \\
\text { (ING) }\end{array}$ & Informática & 51 & 51 & $2 \%$ & $4 \%$ \\
\hline
\end{tabular}

Fuente: Elaboración propia. 


\section{Instrumento}

La recogida de información se realizó a partir del Cuestionario de Evaluación de Competencias Transversales de Grado (CECTGRA) diseñado ad hoc. Este cuestionario integra cinco bloques de preguntas cuantitativas y cualitativas, elaborados todos en torno al bloque $\mathrm{C}$ o escala de competencias que recoge la valoración del desarrollo o grado de enseñanza de las competencias transversales en las aulas universitarias, el dominio o adquisición que presenta el estudiante y la relevancia o valor que se otorga para el desarrollo profesional (Figura 1).

Figura 1 - Estructura del cuestionario CECTGRA

\begin{tabular}{|c|c|c|c|}
\hline \multicolumn{2}{|c|}{ BLOQUE AF datos personales } & \multicolumn{2}{|c|}{$\begin{array}{c}\text { BLOQUE B: experiencia académico y } \\
\text { profesional }\end{array}$} \\
\hline \multicolumn{2}{|c|}{ Facultad, especialidad, sexo y edad } & \multicolumn{2}{|c|}{$\begin{array}{l}\text { Horas de estudio, asistencia a clase, nota media, } \\
\text { métodos de enseñanza, libre configuración, } \\
\text { becas, situación laboral }\end{array}$} \\
\hline & \multicolumn{2}{|c|}{$\begin{array}{l}\text { BLOQUE G: competencias } \\
\text { transversales }\end{array}$} & \\
\hline $\begin{array}{l}\text { - Desarrollo extracurricular de } \\
\text { las CT } \\
\text { - Desarrollo curricular de las } \\
\text { CT y materias } \\
\text { - Relevancia de las CT y la } \\
\text { empleabilidad }\end{array}$ & \multicolumn{2}{|c|}{$\begin{array}{l}\text { - Puntos fuertes } \\
\text { - Puntos débiles } \\
\text { - Propuestas de la mejora }\end{array}$} & $\begin{array}{l}\text { - Satisfacción } \\
\text { - Utilidad y adecuación } \\
\text { - Propuestas de mejora para } \\
\text { ele ajuste competencial }\end{array}$ \\
\hline $\begin{array}{l}\text { BLOQUE D: fatores } \\
\text { curriculares y sociales }\end{array}$ & \multicolumn{2}{|c|}{$\begin{array}{c}\text { BLOQUE Et fortalezas y } \\
\text { debilidades de la formación } \\
\text { en GT }\end{array}$} & $\begin{array}{l}\text { BLOQUE Fł valorización } \\
\text { general de la formación }\end{array}$ \\
\hline
\end{tabular}

Fuente: Elaboración propia.

Para dar respuesta al objetivo de este trabajo nos centraremos en analizar la dimensión dominio del bloque $\mathrm{C}$ y las variables del bloque $\mathrm{B}$ (a excepción de las metodologías de enseñanza utilizadas en el aula, por no ser objeto de este estudio). Todas las respuestas se recogen en una escala de valoración numérica con cinco opciones de respuesta $(1=\mathrm{Nada}-5=$ Mucho $)$.

El listado de competencias transversales que contempla la escala del bloque C fue definido ad hoc por Martínez Clares y González Morga (2018) tras la consulta de nueve documentos clave para el diseño de los títulos de grado y catorce estudios que contemplan la visión de los empleadores sobre las competencias más requeridas en el mercado 
productivo en los últimos años. Esta relación de competencias se detalla en la tabla 2 y han sido agrupadas en función del modelo Tuning, adoptado por Agencia Nacional de Evaluación de la Calidad y Acreditación (ANECA) años más tarde para la elaboración de los libros blancos de las titulaciones universitarias españolas, cuyas competencias se estructuran en instrumentales, interpersonales y sistémicas. Esta estructura interna ha sido validada a través de Análisis Factorial Confirmatorio con unos indicadores de bondad de ajuste satisfactorios (dimensión dominio: NFI=.903; CFI=.916; RMSEA=.074) y comprobada su fiabilidad tanto de la escala global (alfa=.881) como de las subescalas resultantes (competencias instrumentales, alfa=.718; competencias personales, alfa=.774; competencias sistémicas, alfa=.836).

Tabla 2 - Escala de competencias transversales

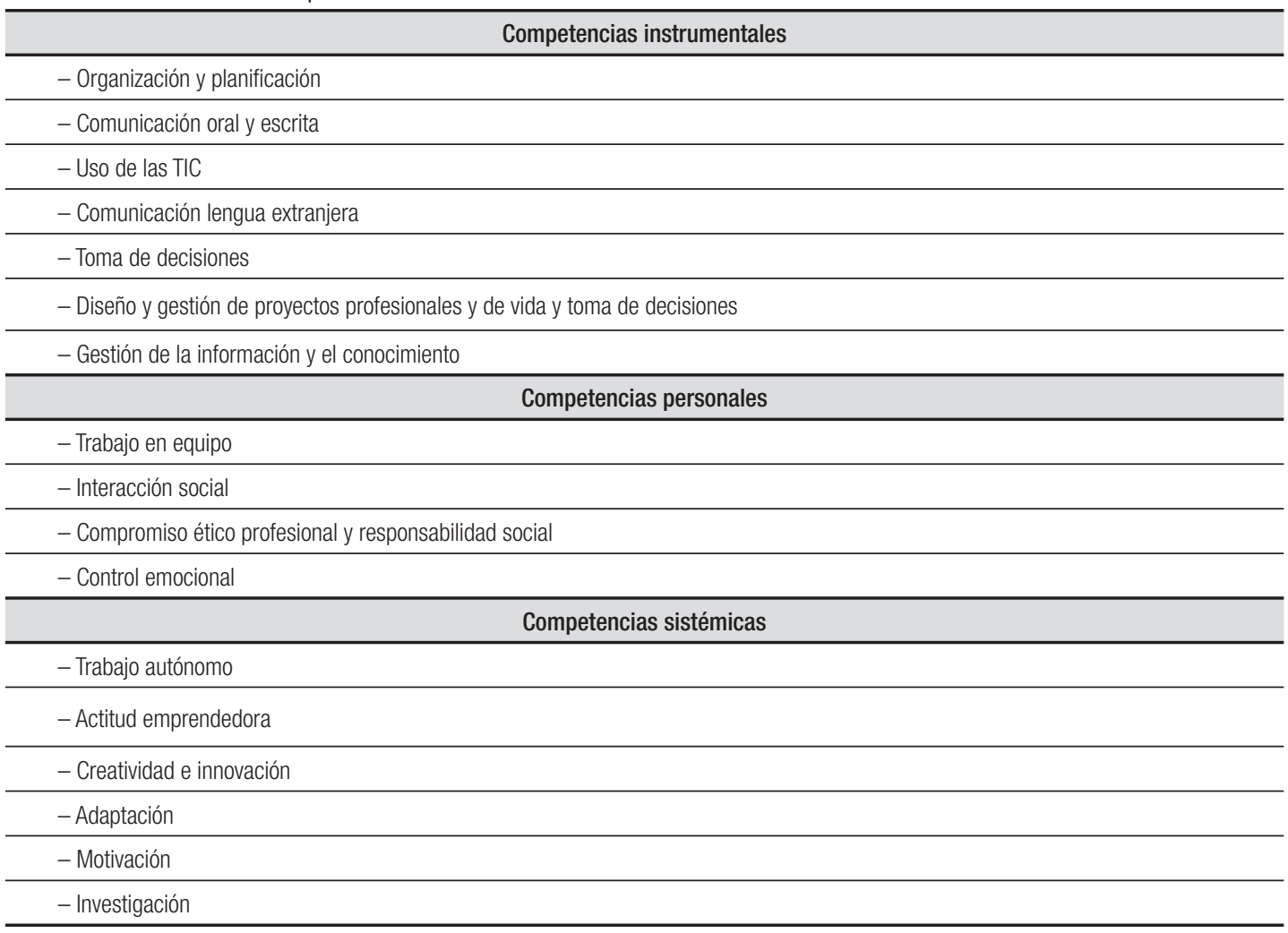

Fuente: Elaboración propia.

\section{Procedimiento}

La recogida de información se planifica en el segundo cuatrimestre del curso 2014/2015, con la intención de que el alumnado tuviera una percepción lo más completa posible de la formación universitaria. Para la aplicación, se contacta con los tutores de 
$4^{\circ}$ de Grado de cada grupo-clase y son los propios miembros del equipo de investigación, quienes se trasladan al aula para realizar una breve explicación del cuestionario e insistir en su carácter voluntario, anónimo y confidencial. Para el análisis de los datos se recurre tanto a la estadística descriptiva (media, desviación típica) como a la inferencial a través de pruebas no paramétricas (Friedman para muestra relacionadas y U de Mann Whitney y $\mathrm{H}$ de Kruskal Wallis para muestras independientes de dos o más grupos respectivamente, así como correlaciones bivariadas a través del estadístico Rho de Spearman), dada la no distribución normal de los datos comprobada con la prueba de Kolmogorov Smirnov. Para el tratamiento de los datos se usa el software estadístico para análisis de datos cuantitativos SPSSv.22.

\section{Resultados}

\section{Dominio del estudiante de Grado en competencias transversales}

El grado de dominio que presenta el estudiante de las competencias transversales es aceptable, si bien mejorable, con un valor medio que oscila en torno al 3,5, siendo la competencia más dominada el compromiso ético y social y la menos el conocimiento de una lengua extranjera (Figura 2). El análisis descriptivo muestra que no todas las competencias se dominan al mismo nivel, existiendo diferencias significativas $\left[X^{2}=4233.906\right.$; gl (15); $\left.p=.000\right]$.

Figura 2 - Análisis descriptivo dominio de las competencias transversales. Media y desviación típica

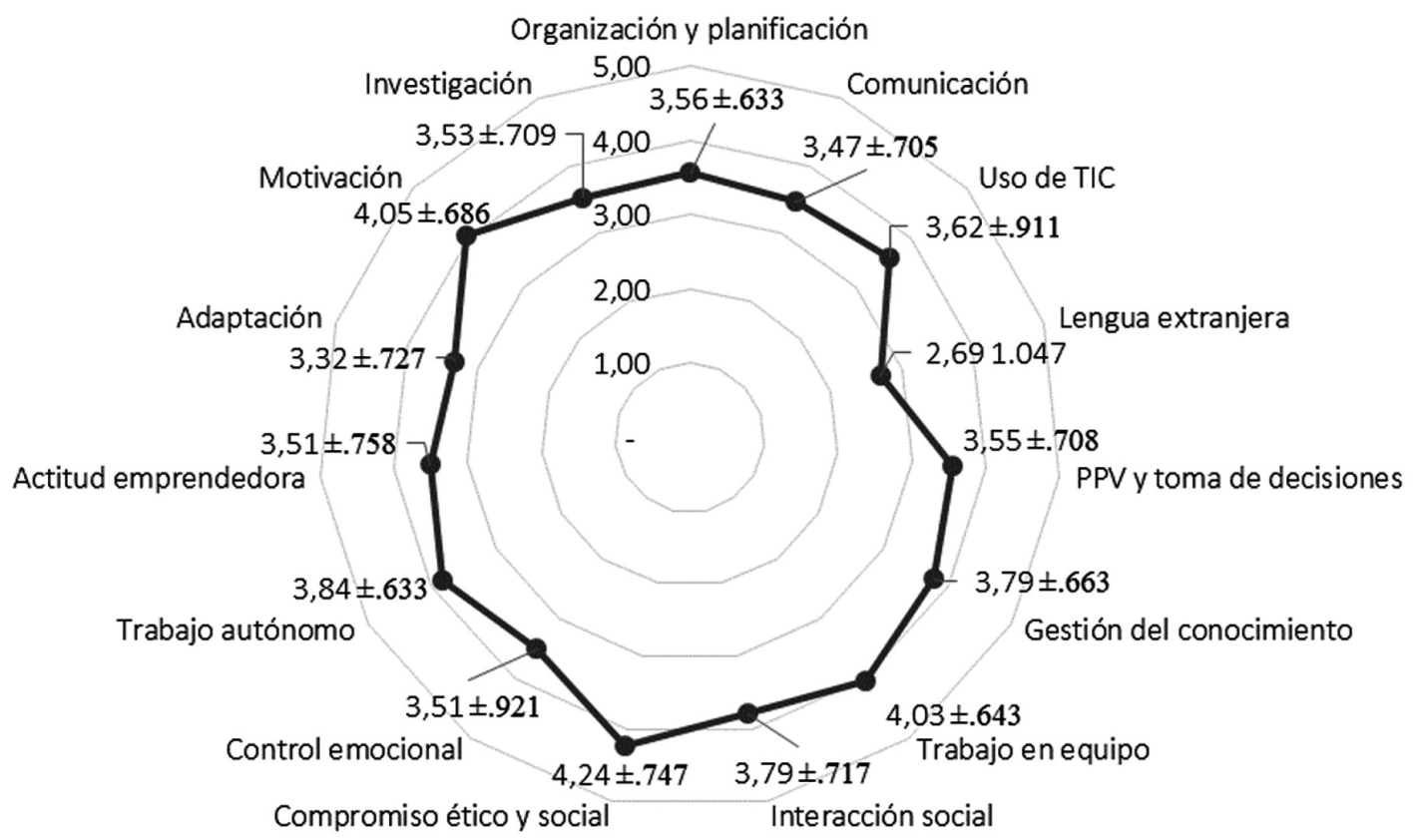

Fuente: Elaboración propia. 
De igual forma existen diferencias entre los estudiantes de las distintas ramas de conocimiento $(p<.03)$. Aunque esta constante de respuesta se repite en CSS e ING (figura 3), siendo en estas ramas de conocimiento donde los estudiantes perciben un dominio más alto, es en CE donde se ha detectado entre el alumnado un dominio de estas competencias más bajo.

Figura 3 - Análisis descriptivo dominio de las competencias transversales por rama de conocimiento- Media

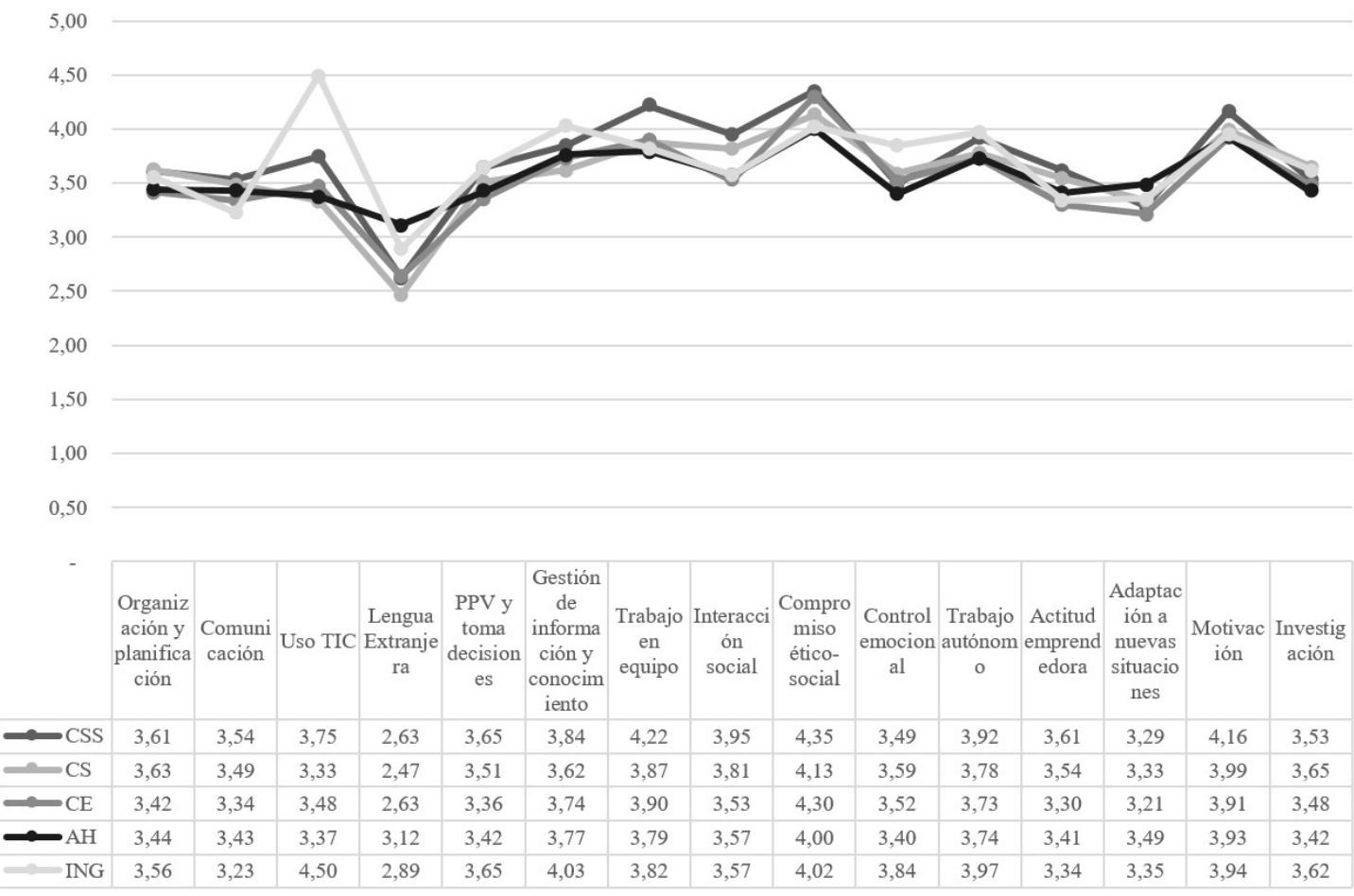

Fuente: Elaboración propia.

Relación entre el dominio de las competencias transversales y la experiencia académica

El dominio difiere en función de la experiencia académica y profesional del estudiante. En primer lugar, se analiza el dominio o grado de adquisición de las competencias transversales en función de diferentes aspectos de su experiencia curricular.

Horas de estudio y dominio de las competencias transversales

Se representa (Figura 4) la estadística descriptiva del dominio de las competencias en relación a las horas que dedica el alumnado al estudio a la semana. 
Figura 4 - Análisis descriptivo del dominio de las competencias transversales en relación a las horas de estudio- Media

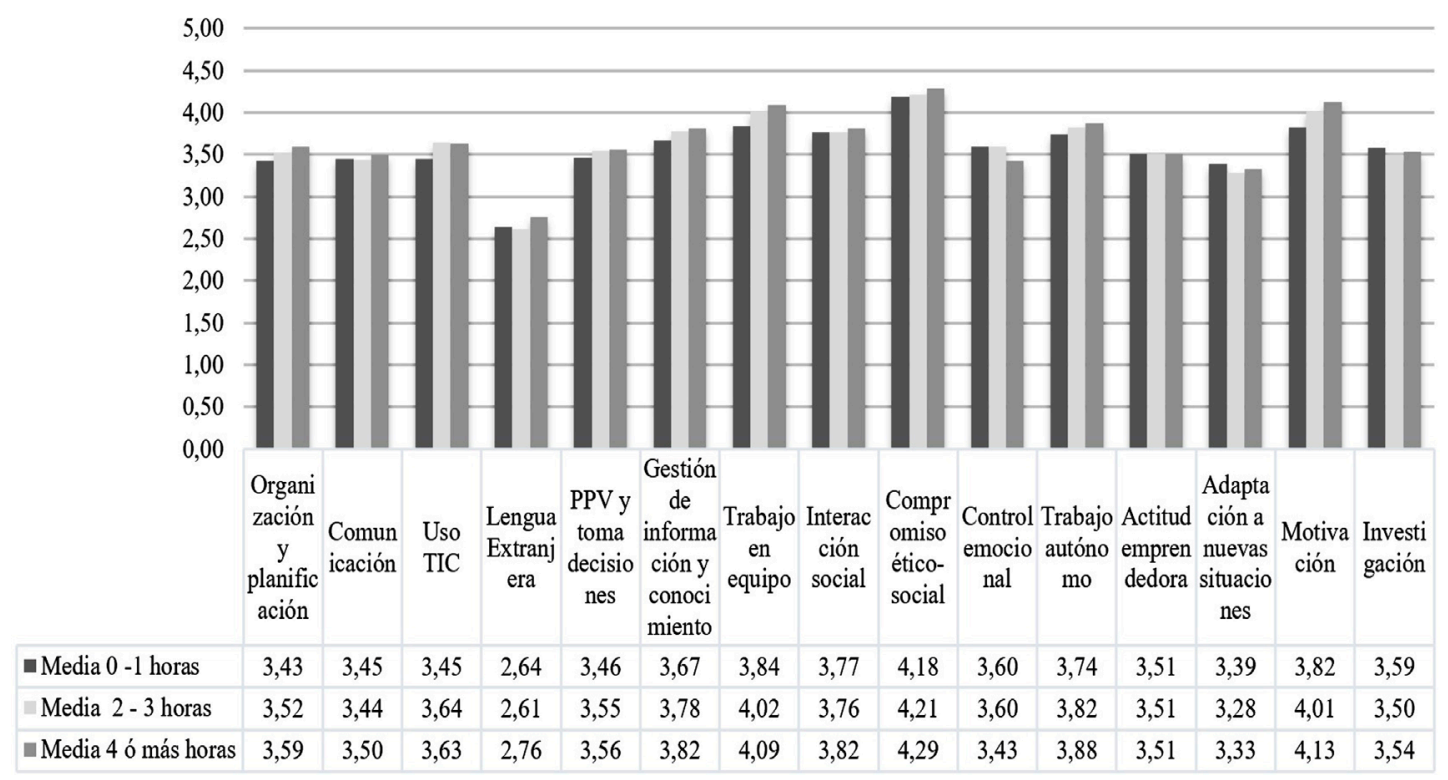

Fuente: Elaboración propia.

El alumno que más horas dedica al estudio es el que obtiene, en prácticamente la totalidad de las competencias, el dominio más elevado, siendo estas diferencias significativas en la competencia de organización y planificación $(p=.020)$, trabajar en equipo $(p=.000)$ y la motivación $(p=.000)$. El hecho de pasar más tiempo dedicado a tareas académicas refuerza su capacidad de organizar, planificar y cooperar en el trabajo, además de potenciar su motivación intrínseca y extrínseca por la tarea bien hecha. Por el contrario, el alumnado que menos tiempo dedica al estudio es el que obtiene la valoración significativamente más alta en control emocional, $(p=.004)$.

\section{Asistencia a clase y dominio de las competencias transversales}

En relación con la asistencia a clase, el análisis descriptivo de la Figura 5 muestra un grado de dominio más elevado en aquellos estudiantes que no asisten a clase con asiduidad, especialmente significativo en la competencia de Interacción social $(p<.035)$, compromiso ético y social $(p<.035)$, actitud emprendedora $(p<.022)$ y motivación $(p<.00)$. Sin embargo, la asistencia a clase parece reforzar la adquisición de la competencia como la lengua extranjera ( $p=.034$ ), percibiéndose un dominio más elevado en aquellos estudiantes que van a clase de forma continuada. 
Figura 5 - Análisis descriptivo del dominio de las competencias transversales en función de la asistencia a clase del estudiante. Media

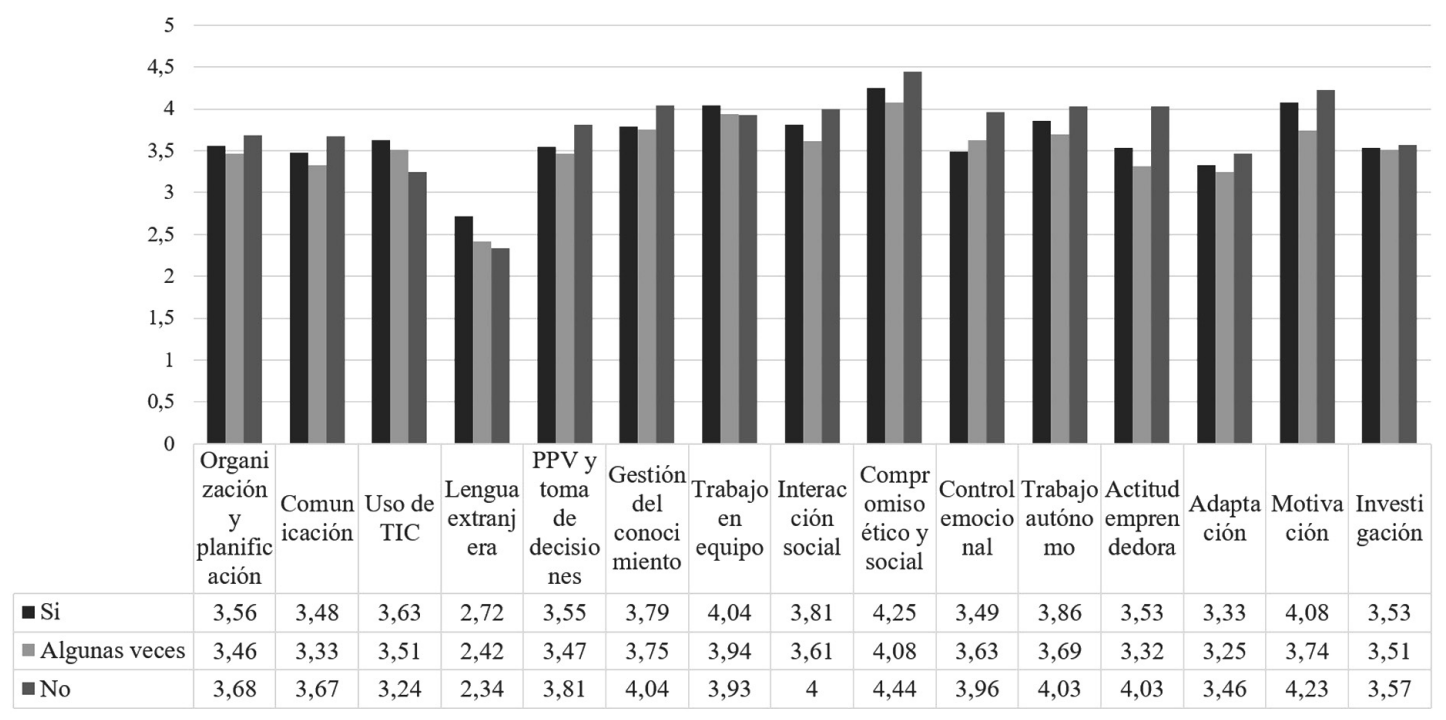

Fuente: Elaboración propia.

Nota media y dominio de las competencias transversales

El dominio de las competencias transversales es superior en aquellos estudiantes que han obtenido calificaciones medias más altas. Las diferencias encontradas en los diferentes grupos establecidos en función de la nota son estadísticamente significativas en prácticamente la totalidad de las competencias analizadas $(\mathrm{p}<.05)$, a excepción de las competencias de control emocional $(\mathrm{p}<.259)$ y compromiso ético y social $(\mathrm{p}<.088)$, aunque siguen el patrón de respuesta de la mayoría de ellas.

Dada la estrecha relación que se observa entre nota de estudio y dominio competencial, se considera pertinente profundizar en este resultado y analizar con mayor profundidad la relación que cada una de estas competencias tiene en la nota final o rendimiento académico del estudiante. Para ello se recurre a las correlaciones bivariadas de cuyo análisis se desprenden los siguientes resultados (ver Tabla 3).

El grado de dominio que presenta el estudiante de las CT correlaciona de forma significativa, directa y positiva con la nota media en todos los casos, a excepción de la competencia del control emocional y compromiso ético y social. Por otro lado, las correlaciones más altas se establecen entre motivación $(r=.207 ; p=.000)$ y organización $y$ planificación $(\mathrm{r}=.170 ; \mathrm{p}=.000)$ con la nota media de la carrera. Por tanto, cuanto mayor es el dominio o adquisición de estas competencias, mejores son las calificaciones de los estudiantes. 
Tabla 3 - Correlaciones entre dominio de las competencias transversales y la nota de expediente académico

\begin{tabular}{|c|c|c|c|}
\hline & & & \\
\hline & Rho de Sperman & Sig. (bilateral) & $\mathrm{N}$ \\
\hline Organización y planificación & $.170^{\star \star}$ & .000 & 1137 \\
\hline Comunicación & $.154^{\star *}$ & .000 & 1137 \\
\hline Uso TIC & $.089^{\star *}$ & .003 & 1137 \\
\hline Lengua Extranjera & $.129^{\star \star}$ & .000 & 1137 \\
\hline PPV y toma decisiones & $.112^{\star *}$ & .000 & 1137 \\
\hline Gestión de información y conocimiento & $.104^{\star *}$ & .000 & 1137 \\
\hline Trabajo en equipo & $.099^{* *}$ & .001 & 1137 \\
\hline Interacción social & $.107^{\star \star}$ & .000 & 1137 \\
\hline Compromiso ético-social & .053 & .052 & 1137 \\
\hline Control emocional & .043 & .147 & 1137 \\
\hline Trabajo autónomo & $.130^{\star \star}$ & .000 & 1137 \\
\hline Actitud emprendedora & $.142^{\star *}$ & .000 & 1137 \\
\hline Adaptación a nuevas situaciones & $.065^{*}$ & .030 & 1137 \\
\hline Motivación & $.207^{\star *}$ & .000 & 1137 \\
\hline Investigación & $.113^{\star *}$ & .000 & 1137 \\
\hline
\end{tabular}

Nota: ${ }^{* *} p<.05$

Fuente: Elaboración propia.

Relación entre el dominio de las competencias transversales y las experiencias extracurriculares y/o profesionales

Una vez analizada la relación de distintos factores académicos en el dominio competencial del alumnado, se contrasta el grado de adquisición con otros aspectos o variables más próximas a la experiencia extracurricular y profesional.

\section{Becas y dominio de las competencias transversales}

Los estudiantes que obtienen una beca de formación en idiomas durante los estudios tienen un dominio superior de las competencias transversales, si bien, estas diferencias son significativas en el conocimiento de una lengua extranjera $(p=.011)$, compromiso ético y social ( $p=.018$ ) y en la adaptación a nuevas situaciones $(p=.014)$. En relación a las becas de movilidad, se perciben diferencias significativas en el dominio que presenta el estudiante que disfruta de estas becas y los que no, en las siguientes competencias (uso de las TIC $(p=.002)$, lengua extranjera ( $p=.000)$, gestión del conocimiento $(p=.030)$, compromiso ético 
$y$ social ( $p=.005)$, trabajo en equipo $(p=.030)$, trabajo autónomo $(p=.016)$, adaptación a nuevas situaciones ( $p=.000)$.

Por último, cabe destacar que los alumnos que disfrutan de becas de investigación tienen un mejor dominio generalizado de las competencias, siendo en el conocimiento de la lengua extranjera donde únicamente se encuentran diferencias estadísticamente significativas $(p=.003)$.

\section{Actividades CRAU y dominio de las competencias transversales}

Se evidencia (Figura 6) un dominio más alto en competencias transversales en aquellos estudiantes que realizan actividades CRAU, siendo estas diferencias estadísticamente significativas en la competencia comunicativa $(\mathrm{p}=.004), P P V$ y toma de decisiones $(p=.017)$, trabajo autónomo ( $p=.012)$ y adaptación a nuevas situaciones $(p=.026)$.

Figura 6 - Análisis descriptivo de la relación dominio y actividades CRAU

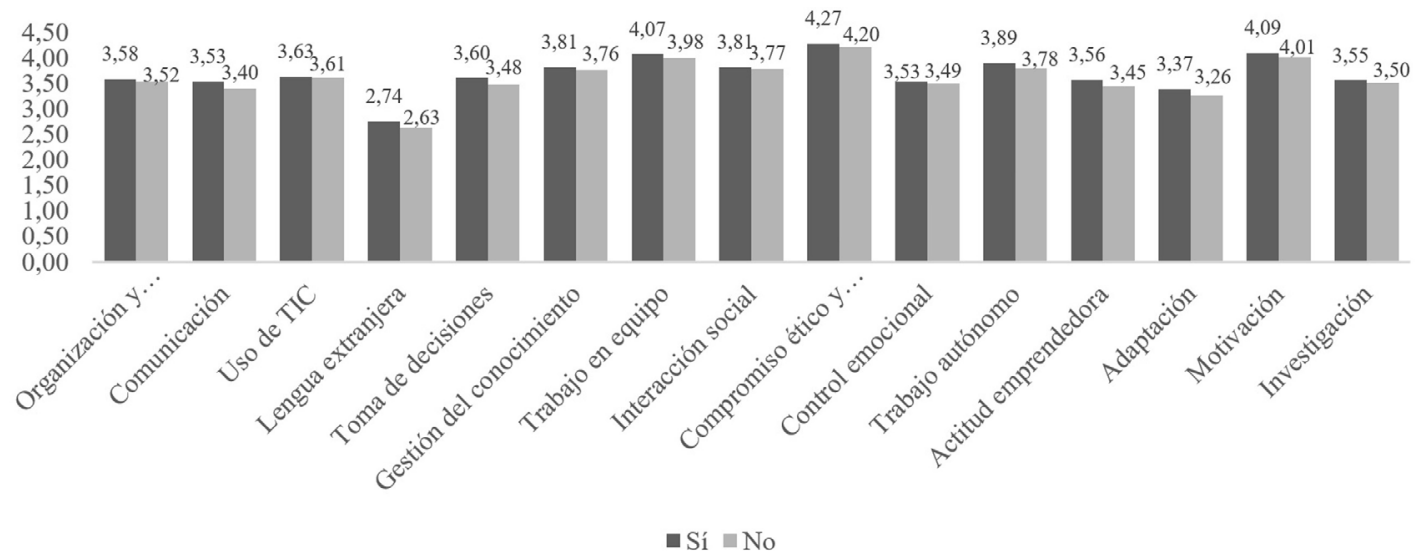

Fuente: Elaboración propia.

Se analizan pormenorizadamente estas diferencias en función del tipo de actividad. En relación a las actividades culturales, el dominio presenta diferencias significativas $(\mathrm{p}<.038)$ en seis de las quince competencias analizadas (comunicación, lengua extranjera, trabajo en equipo, trabajo autónomo, actitud emprendedora y motivación), siendo en todos los casos superior en aquellos estudiantes que las realizan. Por otro lado, el alumnado que participa en actividades deportivas tan solo se encuentra diferencias significativas en la competencia de control emocional, siendo superior en los alumnos que practican alguna de estas actividades ofertadas en la universidad. De igual forma, los estudiantes que realizan actividades de solidaridad y cooperación son más proclives que el resto a adaptarse a nuevas situaciones ( $\mathrm{p}=.05)$. Por último, los estudiantes que desarrollan algún tipo de representación estudiantil presentan un dominio significativamente superior en la mayoría de las competencias transversales. 
Situación laboral del estudiante y dominio de las competencias transversales

La situación laboral que mantiene el estudiante afecta al grado de adquisición de las competencias transversales. Aunque en gran parte de las competencias analizadas, los estudiantes que alternan estudio con trabajo tienen un nivel de dominio similar a los que solo están dedicados exclusivamente a la carrera, es significativamente superior en el diseño del PPV y toma de decisiones ( $p=.021)$; gestión del conocimiento $(p=.007)$; adaptación a nuevas situaciones $(p=.002)$; actitud emprendedora $(p=.000)$ y motivación $(p=.016)$.

Tabla 4 - Análisis descriptivo del dominio de las competencias transversales y diferencias significativas entre grupos

\begin{tabular}{|c|c|c|c|c|c|}
\hline \multirow{2}{*}{ Competencias } & \multicolumn{2}{|c|}{ SOLO ESTUDIA } & \multicolumn{2}{|c|}{ TRABAJA Y ESTUDIA } & \multirow{2}{*}{$\begin{array}{c}\text { U de Mann Whitney } \\
\text { Sig.(p) }\end{array}$} \\
\hline & Media & DT & Media & DT & \\
\hline Organización y planificación & 3.56 & 0.60 & 3.56 & 0.67 & .945 \\
\hline Comunicación & 3.45 & 0.70 & 3.49 & 0.71 & .358 \\
\hline Uso de TIC & 3.63 & 0.90 & 3.61 & 0.93 & .878 \\
\hline Lengua extranjera & 2.69 & 1.02 & 2.70 & 1.08 & .941 \\
\hline Toma de decisiones & 3.51 & 0.70 & 3.60 & 0.71 & .021 \\
\hline Gestión del conocimiento & 3.74 & 0.65 & 3.84 & 0.67 & .007 \\
\hline Trabajo en equipo & 4.02 & 0.64 & 4.04 & 0.64 & .688 \\
\hline Interacción social & 3.77 & 0.72 & 3.82 & 0.71 & .273 \\
\hline Compromiso ético y social & 4.25 & 0.61 & 4.23 & 0.89 & .491 \\
\hline Control emocional & 3.51 & 0.89 & 3.51 & 0.96 & .837 \\
\hline Trabajo autónomo & 3.83 & 0.61 & 3.87 & 0.67 & .280 \\
\hline Actitud emprendedora & 3.42 & 0.75 & 3.63 & 0.76 & .000 \\
\hline Adaptación & 3.26 & 0.70 & 3.40 & 0.75 & .002 \\
\hline Motivación & 4.01 & 0.67 & 4.10 & 0.70 & .016 \\
\hline Investigación & 3.52 & 0.70 & 3.55 & 0.72 & .520 \\
\hline
\end{tabular}

Fuente: Elaboración propia.

El hecho de alternar estudios con trabajo, en ningún caso retrasa la adquisición de estas competencias, sino todo lo contrario, en gran parte de ellas se potencia su dominio por parte del alumnado (Tabla 4).

\section{Conclusiones}

De estos resultados se obtienen las siguientes conclusiones. En primer lugar, los estudiantes próximos a su inserción laboral tienen un nivel de dominio de las CT 
razonable, aunque mejorable especialmente en el área de ciencias experimentales y de forma generalizada en todas las ramas de conocimiento en la competencia de lengua extranjera. Este resultado se reitera en diversos estudios a escala nacional (CONCHADO; CAROT, 2013; MICHAVILA et al., 2016) siendo considerado uno de los principales puntos débiles de la formación universitaria de los egresados. Estas competencias tienen que adquirirlas por otros medios, dada su necesidad para afrontar los retos que plantea una sociedad globalizada sin límites geográficos y donde cada vez más las oportunidades laborales se extienden a la escena internacional.

Salvando esta carencia formativa en lengua extranjera, los resultados son a priori esperanzadores para el ámbito profesional. Estas competencias tienen una alta relevancia en el mercado laboral, en los procesos de selección e inserción profesional, a la vez que los estudiantes parecen tener adquiridas las más significativas. El trabajo en equipo es una de las más dominadas por los estudiantes (MICHAVILA et al., 2016), y es una de las competencias más demandadas por los empleadores (HUMBERG; VAN DER VALDEN; VERHAGEN, 2013; OIT, 2015). Cada vez son más los estudios que ponen de manifiesto la importancia de estas competencias transversales en los profesionales del presente, pero sobre todo del futuro. El concepto de profesionalidad está cambiando y la transversalidad en los perfiles formativo de los graduados se antepone a la especialización para afrontar los empleos cambiantes tanto en término de ubicación y tarea por los que se prevé que pasarán a lo a largo de sus carreras profesionales. Según los últimos informes del WFE (2016), 7,1 millones de empleos se destruirán y 2,1 millones de nuevos empleos se crearán en los próximos años. A pesar de los resultados positivos obtenidos en el dominio de las competencias analizadas, estos indican la necesidad desde las instituciones universitarias de seguir trabajando para reforzar esta formación en paralelo al desarrollo de la situación económica que acontece.

Las líneas de actuación a seguir para reforzar estas competencias entre los estudiantes de Educación Superior se pueden obtener de las siguientes conclusiones de este trabajo. El hecho de un dominio más o menos alto puede deberse a distintos factores, si bien, los resultados de esta investigación constatan una relación significativa entre distintos aspectos de las experiencias curriculares y extracurriculares que alternan los estudiantes durante los estudios. Los resultados muestran un dominio más alto en aquellos estudiantes que dedican más tiempo al estudio, especialmente en organización y planificación, trabajo en equipo y motivación. De igual forma, el dominio de estas competencias es mayor en aquellos estudiantes que obtienen una media de sobresaliente, de forma que cuanto mayor es el dominio de estas competencias mejores son las calificaciones de los estudiantes. De ahí que estas dos competencias puedan considerarse con un desarrollo primordial en la formación universitaria dada la repercusión que su dominio tiene en el expediente académico del estudiante. Si bien, existen excepciones. Los resultados muestran que el hecho de dedicarle más tiempo a la carrera no es un factor que favorece la adquisición de la competencia emocional, por lo que sería pertinente seguir indagando sobre esta cuestión para determinar que otros factores curriculares y extracurriculares podrían favorecerla, dada su relevancia en el mundo sociolaboral. Actualmente, todas las relacionadas con la inteligencia emocional son consideradas esenciales y prioritarias para el trabajador del 
2020 (WEF, 2016). En un entorno productivo cada vez más volátil, incierto, complejo, ambiguo y en plena transición hacia la cuarta Revolución Industrial (WEF, 2016), son necesarios profesionales flexibles y polivalentes capaces de afrontar los cambios con tolerancia y gestión de la incertidumbre, unas capacidades irremplazables por la tendencia a la automatización de las máquinas, a pesar del amplio rango de nuevas funciones y competencias que pueden desempeñar y que hasta ahora eran de exclusividad humana.

Por otro lado, la asistencia a clase parece ser un factor poco relacionado con el incremento del dominio competencial, a excepción de la lengua extranjera. Estudios como los de Michavila y otros autores (2016), Pérez, García y Sierra (2013) o Sánchez Elvira, López González y Fernández Sánchez (2010) ponen de manifiesto el escaso desarrollo de estas competencias en el aula y la necesidad de incrementar su presencia y coherencia en los planes de estudio para una inclusión definitiva en la práctica docente. La OIT (2015) o Mourshed, Patel y Suder (2014) reconocen que estar en posesión de un título universitario no es garantía de tener las competencias adecuadas si durante el periodo formativo no se planifican, se desarrollan y finalmente se adquieren las competencias más genéricas.

Expresada la relación del dominio de las CT con la experiencia curricular del estudiante, se concluye con la necesidad de potenciar las competencias transversales en las aulas para reducir la brecha entre el perfil formativo y el perfil profesional, pero también para favorecer el éxito académico en el estudiante, dado que aquellos estudiantes con mejores calificaciones son los que obtienen el dominio más alto de las mismas. En esta línea, Miró (2010) y Villanueva (2014) percibían también las CT, no solo como una opción estratégica para el éxito laboral, sino también para mejorar su rendimiento académico.

La universidad no es la única responsable de la adquisición de estas competencias por parte del estudiante. Concretamente, aquellos que dicen haber disfrutado de alguna beca de formación de idiomas, movilidad o de investigación, obtienen un dominio más elevado de las competencias analizadas, y significativamente más alto de las competencias de lengua extranjera y adaptación a nuevas situaciones. Lo mismo ocurre con los estudiantes que han alternado los estudios con algún trabajo, siendo el dominio significativamente superior en toma de decisiones, gestión del conocimiento, actitud emprendedora, adaptación a nuevas situaciones y motivación. En el análisis que realiza ANECA (2007) se obtienen resultados similares, con una correlación positiva entre el dominio de los estudiantes y la participación en actividades complementarias de forma general, y en particular en programas de movilidad o que han alternado estudios con trabajo, siendo especialmente relevantes para el dominio del idioma extranjero y la capacidad de comunicarse o hablar en público.

Las respuestas de los estudiantes parecen indicar que las instituciones de Educación Superior deban impulsar una mayor oferta de programas internacionales, de investigación y de formación como estrategia pedagógica para sumar experiencias positivas que favorecen la adquisición de competencias transversales. A esta misma conclusión llega Rodríguez Izquierdo (2015) tras contrastar el impacto de los proyectos internacionales en el dominio de estas competencias en los estudiantes universitarios. Concretamente los estudiantes reconocen haber cambiado la forma de orientar su desarrollo profesional y a la vez ampliado sus perspectivas de trabajo futuro en un entorno internacional, de 
forma que aquellos que han disfrutado de periodos en el extranjero sienten que han adquirido una formación más integral, que han tomado conciencia de sus propios prejuicios y estereotipos y se sienten más capaces de trabajar y desenvolverse en contextos internacionales. La participación en estos proyectos supone un cambio de actitud en los egresados en la forma de orientar el desarrollo académico y profesional que amplía las perspectivas y oportunidades de trabajo al entorno internacional.

Además de los programas de movilidad, se ha valorado la repercusión de la realización de otras actividades formativas o de investigación paralelas al currículo universitario en el bagaje de competencias transversales del estudiante, tales como deportivas, de realización de voluntariado o de representación estudiantil dentro de las CRAU. El Centro de Orientación e Información de Empleo (COIE, 2007) de la Universidad objeto de análisis, consideró a la formación complementaria como uno de los principales medios de adquisición de competencias trasversales, junto con las prácticas externas. Además, según el Observatorio de Innovación en el Empleo, los propios universitarios consideran "[...] la formación complementaria un valor añadido y un elemento diferencial para competir en el mercado laboral” (OIE, 2014, p. 32). Sin embargo, aunque la mayoría es consciente de la importancia de esta formación, no se suele tener en cuenta hasta los últimos años de carrera, según estos informes.

Asimismo, a las experiencias internacionales y formativas hay que añadir las procedentes del ámbito profesional. La adquisición de competencias de los estudiantes que combinan la carrera con trabajos es superior en todas las competencias y especialmente relevante para favorecer la toma de decisiones, adaptarse a nuevas situaciones y mostrar una actitud más emprendedora y con mayor motivación por el trabajo. Estos resultados concuerdan con los obtenidos por De la Iglesia (2011), Clemente y Escribá (2013) y Corominas (2001) percibiendo en todos los casos un mayor dominio de estas competencias en los estudiantes que tienen experiencia profesional. Según el estudio realizado por Ruesga, Da Silva y Monsueto (2014), existe un doble efecto a considerar en el impacto del trabajo del estudiante sobre el desempeño académico. El primero consiste en que los estudiantes que trabajan o alternan la formación con otras actividades extracurriculares pueden presentar mejores resultados académicos al establecerse una relación complementaria entre formación académica y experiencia sociolaboral, existiendo un número de horas semanales dedicadas al trabajo a partir del cual se observan efectos negativos sobre el desempeño académico. El segundo, afirma que la experiencia laboral previa a la entrada en la universidad contribuye a mejorar el desempeño académico. En cualquier caso, con estas interpretaciones y resultados obtenidos se reflejan "[...] la necesidad de políticas específicas dirigidas a aquellos estudiantes más jóvenes, sin experiencia laboral y que deciden estudiar y trabajar al mismo tiempo" (CES, 2015, p. 178) dada su repercusión en el dominio competencial.

Cada vez son más los universitarios que optan por compatibilizar los estudios con el trabajo según la OCDE (2013) y la Encuesta de condiciones de vida y participación de los estudiantes universitarios (ECOVIPEN) (ARIÑO; LLOPIS; SOLER, 2012), siendo hasta el momento, una cuestión importante, novedosa y escasamente analizada. Esta 
investigación pretende resultar un avance en este sentido y poner de manifiesto su impacto en el dominio de las competencias transversales. De esta forma, se evita caer en la interpretación reduccionista que ya advertían Planas Coll y Enciso Ávila (2014), de responsabilizar únicamente la formación curricular de la adquisición de competencias en los egresados. La realidad inevitablemente es más compleja y mucho tiene que ver otras actividades o experiencias extracurriculares que han simultaneado los estudiantes durante sus estudios.

Las propias características de las competencias transversales se asocian a una amplia diversidad de posibilidades educativas que desborda a las instituciones superiores. Su aprendizaje por parte de los estudiantes no solo se debe a la enseñanza formal, sino que resulta importante tener en cuenta la procedente del ámbito no formal e informal como la impulsada por las becas, actividades universitarias, la experiencia profesional e incluso las relaciones sociales. Con esta información se entiende la adquisición de competencias como un proceso dinámico que requiere de variedad de acciones sistematizadas, integradas y paralelas al currículo universitario para obtener el máximo rendimiento. La alternancia de contextos formativos durante la trayectoria universitaria supone una experiencia formativa entre la esfera educativa y profesional que propicia la continuidad de formación en una discontinuidad de espacios y de tiempo, como afirma Tejada (2012), con repercusiones directas en la profesionalidad de los egresados. Todo ello favorece un nuevo espacio en el que resulta necesario adaptar los procesos e itinerarios formativos considerando la formación en competencias transversales como un eje imprescindible para una favorable inserción sociolaboral.

\section{Referencias}

ANECA. Agencia Nacional de Evaluación de la Calidad Universitaria y Acreditación. Informe ejecutivo. E profesional flexible en la Sociedad del Conocimiento. Madrid: ANECA, 2007.

ANECA. Agencia Nacional de Evaluación de la Calidad Universitaria y Acreditación. Libro blanco para los títulos de grado. Madrid: ANECA, 2005.

ARIÑO, Antonio; LLOPIS, Ramón; SOLER, Inés (Ed.). Desigualdad, diversidad y universidad: condiciones de vida y de participación de los estudiantes universitarios en España (EcoVipeu 2012). Valencia: Observatorio de la Vida y Participación de los Estudiantes, 2012. Disponible en: <https://www.acade mia.edu/3638423/ Desigualdad_diversidad_y_Universidad>. Acceso en: 12 oct. 2017.

CEDEFOP. Centro Europeo para el Desarrollo de la Formación Profesional. Desajuste de competencias: más de lo que parece a simple vista. Nota informativa. Salonica: Cedefop, 2014. Disponible en: <https://www. sepe.es/contenidos/personas/formacion/refernet/pdf/NI-032014_es.pdf>. Acceso en: 5 my. 2015.

CES. Consejo Económico y Social. Informe competencias profesionales y empleabilidad. Madrid: CES, 2015.

CLEMENTE, José; ESCRIBÁ Carmen. Análisis de la percepción de las competencias genéricas adquiridas en la Universidad. Revista de Educación, Madrid, n. 362, p. 535-561. D0l:10.4438/1988-592XRE-2013-362-241 
COBO, Cristóbal; MORAVEC, John. Aprendizaje invisible: hacia una nueva ecología de la educación. Barcelona: UBe, 2011.

COIE. Centro de Orientación e Información de Empleo. Informe de evaluación para el desarrollo de competencias profesionales y su influencia en la inserción laboral. Murcia: COIE: Universidad de Murcia, 2007.

CONCHADO, Andrea; CAROT José Miguel. Puntos fuertes y débiles en la formación por competencias según los graduados universitarios españoles. Revista de Docencia Universitaria - REDU, Valencia, v. 11, n. 1, p. 429-446, 2013.

COROMINAS, Enric. Competencias genéricas en la formación universitaria. Revista de Educación, Madrid, n. 325, p. 299-321, 2001.

CROWLEY, Lizzie et al. International lessons: youth unemployment in the global context. Lancaster: The Work Foundation, 2013.

DE LA IGLESIA, María Covadonga. Adecuación del grado de desarrollo de la formación en competencias a la necesidad en el entorno laboral, según la opinión de los estudiantes. Revista Complutense de Educación, Madrid, v. 22, n. 1, p. 71-92, 2011. DOl: 10.5209/rev_RCED.2011.v22.n1.4

FINKEL, Lucila; BARAÑANO, Margarita. La dedicación al estudio y al trabajo de los estudiantes universitarios en España. Revista de la Asociación de Sociología de la Educación, Valencia, v. 7, n. 1, p. 82-103, 2014.

HUMBERG, Martin; VAN DER VALDEN, Rolf; VERHAGEN, Annelore. The employability of higher education graduates: the employers' perspective. Maastricht: Oficina de Publicaciones de la Unión Europea, 2013.

LE BOTERF, Guy. De la competénce: essai sur un attracteur étrange. París: Les Editions d'Organisations, 1995.

LEVY LEBOYEC, Claude. Gestión de las competencias: cómo analizarlas, cómo evaluarlas, cómo desarrollarlas. Barcelona: Gestión 2000, 1997.

MARTíNEZ CLARES, Pilar; GONZÁLEZ MORGA, Natalia. Las competencias transversales en la universidad: propiedades psicométricas de un cuestionario. Educación XXI, Madrid, v. 22, n. 1, p. 262, 2018. D0l:10.5944/educxx1.15662

MICHAVILA, Francisco et al. Barómetro de empleabilidad y empleo de los universitarios en España, 2015. Madrid: Observatorio de Empleabilidad y Empleo Universitarios, 2016. Primer informe de resultados.

MIRÓ, Joe. Para qué sirven las competencias transversales. Islas Baleares: Universidad Islas Baleares, 2010. Disponible en: <http://bioinfo.uib.es/ joemiro/CTens/PorqueCT.pdf>. Acceso en: 14 oct. 2016.

MOURSHED, Mona; PATEL, Jigar; SUDER, Katrin. Education to employment: getting Europe's youth into work. Washington, DC.: McKinsey, 2014. 
NILSSON, Bjorn. Does the work-study combination among youth improve the transition path? Work4youth technical brief. Ginebra: OIT, 2015.

OCDE. Organización para la Cooperación y el Desarrollo Económicos. Panorama de la educación 2013: nota de España. París: OCDE, 2013. Disponible en: <https://www.oecd.org/edu/Spain_EAG2013\%20 Country\%20Note\%20(ESP).pdf>. Acceso en: 15 oct. 2016.

OIE. Observatorio de Innovación en el Empleo. Informe OIE sobre jóvenes y mercado laboral: el camino del aula a la empresa. Madrid: OIE, 2014. Disponible en: <http://www.oie.es/wp-content/uploads/2015/09/ oie_estudio.pdf>. Acceso en: 12 oct. 2016.

OIT. Organización Internacional del Trabajo. Tendencias mundiales del empleo juvenil 2015: promover la inversión en empleos decentes para los Jóvenes. Ginebra: OIT, 2015.

PÉREZ, Jorge Enrique; GARCÍA, Javier; SIERRA, Almudena. Desarrollo y evaluación de competencias genéricas en los títulos de grado. Revista de Docencia Universitaria, Valencia, v. 11, n. esp. p. 175-196, 2013.

PLANAS COLL, Jordi; ENCISO ÁVILA, Isabel María. Los estudiantes que trabajan: ¿tiene valor profesional el trabajo durante los estudios? Revista Iberoamericana de Educación Superior, México, DF, v. 5, n. 12, p. 23-45, 2014.

RODRÍGUEZ IZQUIERDO, Rosa María. Competencias genéricas en la enseñanza superior a través de los programas de internacionalización. Revista Complutense de Educación, Madrid, v. 26, n. 1, p. 81-100, 2015. D0I: 10.5209/rev_RCED.2015.v26.n1.42598

RUESGA, Santos; DA SILVA, Julimar; MONSUETO, Sandro Eduardo. Estudiantes universitarios, experiencia laboral y desempeño académico en España. Revista de Educación, Madrid, n. 365, p. 67-95, 2014. D0I: 10.4438/1988-592X-RE-2014-365-265

RYCHEN, Dominique Simone; SALGANIK, Laura Hersh (Ed.). Key competencies for a successful life and well-functioning society. Gotinga: Hogrefe \& Huber, 2003.

SÁNCHEZ ELVIRA, Ángeles; LÓPEZ GONZÁLEZ, María Ángeles; FERNÁNDEZ SÁNCHEZ, María Virginia. Análisis de las competencias genéricas en los nuevos títulos de grado del EEES en las universidades españolas. Revista de Docencia Universitaria, Valencia, v. 8, n. 1, p. 35-73, 2010.

SCHWAB, Klaus. The fourth industrial revolution. Ginebra: World Economic Forum, 2016.

TEJADA, José. La alternancia de contextos para la adquisición de competencias profesionales en escenarios complementarios de educación superior: marco y estrategia. Educación XXI, Madrid, v. 15, n. 2, p. 17-40, 2012. DOI: 10.5944/educxx1.15.2.125

VILLA, Aurelio; POBLETE, Manuel. Evaluación de competencias genéricas: principios, oportunidades y limitaciones. Bordón, Madrid, v. 63, n. 1, p. 147-170, 2011. 
VILLANUEVA, Gloria. Competencias genéricas en estudiantes universitarios: elaboración y validación de un instrumento para la mejora de la calidad universitaria. 2014. 228 p. Tesis (Doctorado) - Facultad de Educación, Universidad Complutense de Madrid, Madrid, 2014. Disponible en: <http://eprints.ucm.es/ 27694/1/T35496.pdf>. Acceso en: 2 febr. 2017.

WEF. Work Economic Fourm. The future of jobs employment, skills and workforce strategy for the Fourth Industrial Revolution. Ginebra: WEF, 2016. Disponible en: <http://www3.weforum.org/docs/WEF_Future_ of_Jobs.pdf>. Acceso en: 5 my. 2017.

ZABALZA, Miguel Ángel. El practicum y las prácticas en empresas en la formación universitaria. Madrid: Narcea, 2013.

Recibido en: 28.11.2017

Aprobado en: 25.04.2018

Pilar Martínez Clares es doctora en Educación y profesora titular del Departamento de Métodos de Investigación y Diagnóstico en Educación.

Natalia González Morga es doctora en Educación y profesora asociada del Departamento de Métodos de Investigación y Diagnóstico en Educación. 